\title{
Using Few Radon Projections to Recover 3-D NMR Spectrum
}

\author{
Fawaz Hjouj \\ Department of Mathematics, The Petroleum Institute, Khalifa University of Science and \\ Technology, United Arab Emirates. \\ E-mail: fhjouj@pi.ac.ae.
}

Received: September 19, 2017/ Accepted: November 26, 2017

\begin{abstract}
Given a regular binary function $f$ on $\mathbf{R}^{2}$ with compact support $D$, we use translation to form a new binary function $g$ from $f$ so that the image representation of $g(x, y)$ is made up of non-overlapping copies of $D$. Thus, $g$ is made up of discrete entities that are surrounded by regions of space. We devise a procedure that can determine the translation parameters using a minimum number of Radon projections $g_{\varnothing}^{\vee}$ of $g$. This model is a mathematical abstraction of an application of the Radon transform in Spectroscopy.
\end{abstract} Keywords: Radon transforms, transformation of an image, Back projection. 


\section{INTRODUCTION}

In [13], we addressed the problem of linear transformation of an image. In this paper, we consider a similar problem of translation, namely: we present a straightforward mathematical model of using few Radon projections to recover a two-dimensional nonnegative function $g(x, y)$ whose image representation is made up of discrete entities surrounded by regions of space. A complete mathematical description of this model is given in the next section. This model is a resembling and a mathematical abstraction of an application of the Radon transform in NMR spectroscopy [1-9]. A survey on the subject is presented in [12].

Fundamentally, we may think of a 3-D NMR spectrum as being made up of tiny bright "stars". The reconstruction problem would then be simpler than anatomical imaging (e.g. as a patient in a CAT scanner). The conventional NMR methodology is severely affected by the long duration of measurements that can sometimes take days [1], [2], and [3], where the objects being investigated may be unstable; experiencing some dynamic behavior or a chemical exchange is taking place...etc.

Perhaps the realizations that the traditional reconstruction method is not the most efficient way has led many authors to introduce alternative methods that are generally related to Radon transform: Kupce and Freeman [1], [2], [3], Kim and Szyperski [4], Kozminski and Zhukov [5], and many others including [7-9]. In [11], three methods were reviewed and a forth method is proposed.

In particular, [1], [2], [3] used the Back-projection in their reconstruction scheme. This scheme can reconstruct a 3-D spectrum by processing one plane at a time. In a given plane, two orthogonal projections are measured and then back projected in the image space. This produces peaks of all conceivable locations in question. A tilted projection would then refine the solution 
further. Figure1 explains this idea as was introduced in [1], [2], [3] which stated that the discrete nature of the NMR resonances implies a rapid convergence. Also, they admit that more projections may be needed for crowded spectra, and that if the two orthogonal projection produced $N$ and $M$ peaks respectively, then we have $N+M$ equations of $N M$ unknown which can be a notoriously underdetermined system (if $N$ and $M$ are large).

As said earlier, our model is a mathematical idealization of this problem, in which we show that two projections are still sufficient to find all conceivable locations without using back projections. We then subject these potential solutions to further tests using other projections. Indeed, the Back-projection approach is not always able to find these locations as was claimed in [1] and shown in Figure 1. For example, Fig $2 d$ is the Back-projection image for the image shown in Fig2a using only two projections (with $\phi=0, \frac{\pi}{2}$ ), which are shown in Fig $2 \mathrm{~b}$ and $2 \mathrm{c}$.

Fig 2e, however, is the Back-projection image using 20 projections. We organize this paper as follows: in Section 2 we build our model of a two-dimensional spectrum. In Section 3, we present a mathematical solution that we devised to recover a spectrum using a few Radon Projections. In Section 4, we present further discussion and remarks. We conclude this paper with our conclusion. In the last part of this introduction we introduce notation and basic tools that will be used in this work: Given a suitable real valued function $f$ on the plane $R^{2}$, we will write the Radon transform of $f$ in the ordinary form [10]:

$$
f_{\varnothing}^{\vee}(p)=\int_{-\infty}^{\infty} f(p \cos \varnothing-s \sin \varnothing, p \sin \varnothing+s \cos \varnothing) d s
$$

Among the known properties of this transform we recall the translation rule:

If $g(x, y)=f\left(x-x_{0}, y-y_{0}\right)$, where $x_{0}$ and $y_{0}$ are real scalars, then 
$g_{\varnothing}^{\vee}(p)=f_{\varnothing}^{\vee}\left[p-\left(x_{0} \cos \varnothing+y_{0} \sin \varnothing\right)\right]$

A common elementary function that we are using in this work is the unit cylinder:

$c(x, y):=\left\{\begin{array}{l}1 \text { if } x^{2}+y^{2} \leq\left(\frac{1}{2}\right)^{2} \\ 0 \text { otherwise }\end{array}\right.$

Using (1) we find

$c_{\varnothing}^{\vee}(p)= \begin{cases}\sqrt{1-4 p^{2}} & \text { if }|p|<\frac{1}{2} \\ 0 & \text { otherwise }\end{cases}$

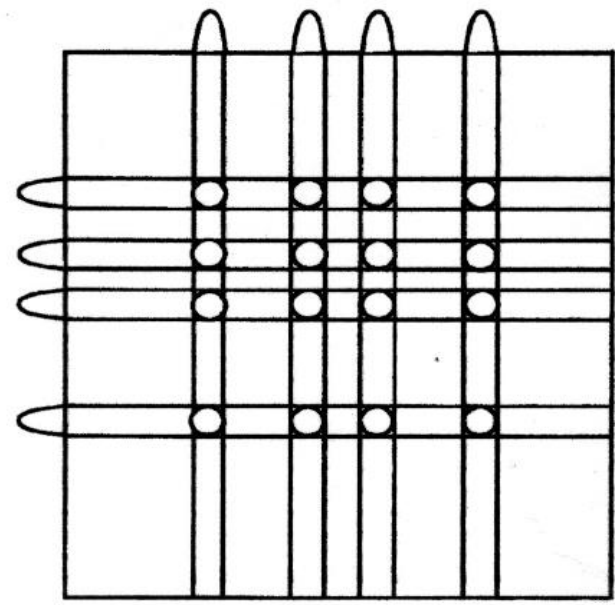

When a two-dimensional NMR spectrum shows four projected peaks on each of two orthogonal axes, there are 16 potential cross-peak positions (circles). But because the problem is underdetermined, some of these may be false cross-peaks (zero intensity). Further information is required to solve the problem unambiguously.

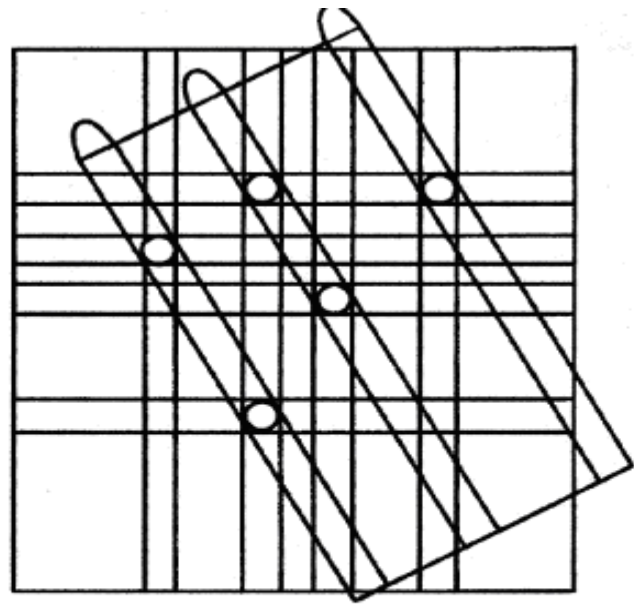

A tilted projection

indicates that 11 of the potential cross-peaks are false because they are not compatible with this new projection. In principle, further tilted projections might be required to confirm the validity of the remaining five peaks, but in practice the problem converges very rapidly.

Figure 1. Schematic diagrams showing the "Superposition of Back-projection" introduced in [1], [2], and [3]. 


\section{A Model of Two-Dimensional Spectrum}

Let $f$ be a regular binary function on the plane $\mathrm{R}^{2}$ with compact support $D$ :

$f(x, y)= \begin{cases}1 & \text { if }(\mathrm{x}, \mathrm{y}) \in \mathrm{D} \\ 0 & \text { otherwise }\end{cases}$

We assume that the center of mass of $\mathrm{D}$ coincides with the origin $(0,0)$. We use $f$ to define a family of binary images of the form

$g(x, y)=\sum_{k=1}^{K} f\left(x-x_{k}, y-y_{k}\right)$

where $\left(x_{1}, y_{1}\right), \ldots,\left(x_{K}, y_{K}\right)$ are pairs of positive real numbers, such that the image

representation of $g(x, y)$ is made up of non-overlapping copies of $\mathrm{D}$. With $f$ in hand, we address the question of recovering $g(x, y)$ using a minimum number of projections $g_{\varnothing}^{\vee}$. Notice that using (2) we write

$g_{\phi}^{\vee}(p)=\sum_{k=1}^{K} f_{\phi}^{\vee}\left[p-\left(x_{k} \cos \phi+y_{k} \sin \phi\right)\right]$

It is possible to assume that $x_{1} \leq x_{2} \ldots \leq x_{K}$ and $y_{1} \leq y_{2} \ldots \leq y_{K}$. We will write any solution $\left(x_{1}, y_{1}\right) \ldots\left(x_{K}, y_{K}\right)$ (that is consistent with (6)) in the form $\boldsymbol{\omega}=\left[\begin{array}{ccccc}x_{1} & x_{2} & \ldots & x_{K} \\ y_{1} & y_{2} & \ldots & y_{K}\end{array}\right]$

We show an example in Fig 2a, where the function $g(x, y)$ is formed using (6), with $f(x, y)$ is the unit cylinder $(3)$ and the parameters $\left(x_{k}, y_{k}\right)$ are as follows:

$(1,6),(1,4.5),(1,2.1),(1.5,3),(2.2,5.5)(2,2),(2.2,4),(3,1.5),(3,2.6),(3.5,5.5),(3.7,3.5),(4$, $4.5),(4.1,2),(5,4),(5,6),(5.5,3),(7, .5)$. 


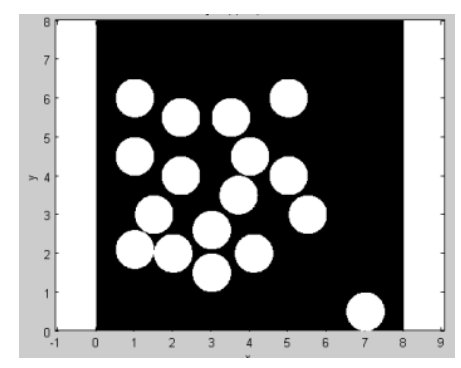

(a)

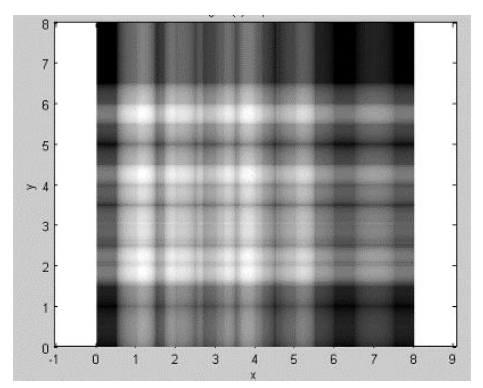

(d)

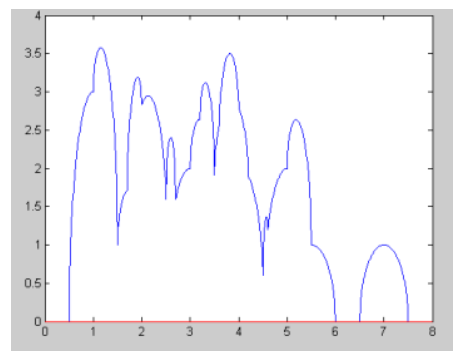

(b)

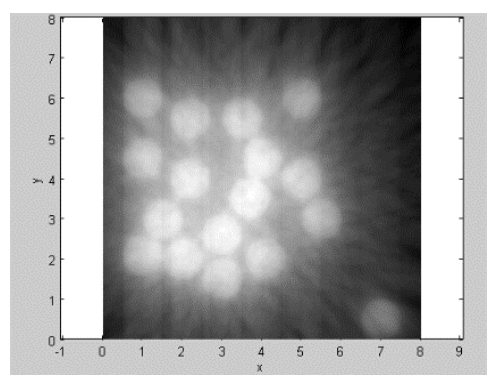

(e)

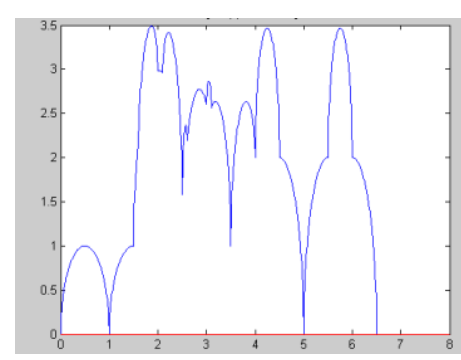

(c)

Figure (2). An example of $g(x, y)$ formed from 17 non- overlapping translations of the unit cylinder (3). (b), (c) show $g_{0}^{\vee}$ and $g_{\frac{\pi}{2}}^{\vee}$ of $g(x, y)$ in (a). (d) Density plot of the Backprojection image of $g(x, y)$ using two projections. (e) Density plot of the Back-projection image of $g(x, y)$ using 20 projections.

\section{Recovering a Spectrum Using Few Radon Projections}

We want to determine the parameters $\left(x_{k}, y_{k}\right)$ that are involved in equation (6). We want to accomplish this goal using $f_{\varnothing}^{\vee}$ and $g_{\varnothing}^{\vee}$ with the minimum number of projections $g_{\varnothing}^{\vee}$. We devised a method to recover these parameters that we now describe. Observe from (7) that

$$
K=\frac{\int_{-\infty}^{\infty} g_{0} \vee(p) d p}{\int_{-\infty}^{\infty} f_{0}^{\vee}(p) d p}
$$

We first show that the parameters $x_{1} \leq x_{2} \ldots \leq x_{K}$ can be obtained using $f_{0}^{\vee}$ and $g_{0}^{\vee}$. 
We also utilize $f_{\frac{\pi}{2}}^{\vee}$ and $g_{\frac{\pi}{2}}^{\vee}$ to produce a list of numbers

$L_{\frac{\pi}{2}}: \quad y_{1} \leq y_{2} \quad \ldots \leq y_{K}$

that are consistent with (6). To find $x_{1}, x_{2}, \ldots, x_{K}$, assume that the support of $g_{0}^{\vee}$ is contained in some closed interval $I_{0}$. Let $v=\left(v_{1}, v_{2}, \ldots, v_{K}\right)$ be a vector of $K$ components with $v_{k} \in I_{0}$ and define the real-valued function $g_{v}(x)$ on $I_{0}$ such that

$g_{v}(x)=\sum_{k=1}^{K} f_{0}^{\vee}\left(x-v_{k}\right)$

In view of these considerations, we defined the objective real-valued function $G$ on the $K$-space $I_{0}{ }^{K}$ such that

$$
\begin{aligned}
G(v)=\| \mathrm{g}_{0}{ }^{\vee} & -\mathrm{g}_{\mathrm{v}} \|_{1} \\
& =\int_{I_{0}}\left|g_{0}{ }^{\vee}(x)-g_{v}(x)\right| \mathrm{dx}
\end{aligned}
$$

Notice that if $v$ is formed using the numbers $x_{1}, x_{2}, \ldots, x_{K}$ from equation (6) then, $G(v)=0$. We can numerically find $\mathrm{v}$ in $I_{0}{ }^{K}$ that minimizes $G$ (e.g. using Nelder-Mead Simplex Method). Since $g_{0}^{\vee}$ is obtained experimentally, we may write

$$
G(v)=\sum_{x}\left|\mathrm{~g}_{0}{ }^{v}(x)-\mathrm{g}_{\mathrm{v}}(x)\right|
$$

where $x$ values are the $x$-coordinates from the sample of the known $g_{0}^{\vee}$. 
To find the list $L_{\frac{\pi}{2}}$ in (9), we simply repeat the minimization process using equations (10) and (11) with $g_{0}^{\vee}$ is replaced by $g_{\frac{\pi}{2}}^{\vee}$ and $f_{0}^{\vee}$ is replaced by $f_{\frac{\pi}{2}}^{\vee}$. With the parameters $x_{1}, x_{2}, \ldots, x_{K}$, and $L_{\frac{\pi}{2}}$ are in hand, we work with the family $\Omega$ of all possible solutions:

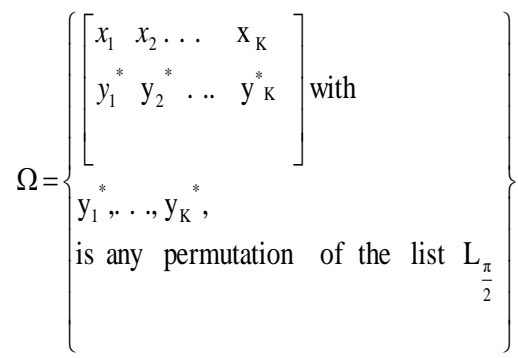

Notice that the unique solution of our problem exists in $\boldsymbol{\Omega}$. Consider a third projection, $g_{\varnothing}^{\vee}$ of $g(x, y)$, we use $g_{\varnothing}^{\vee}$ to test members of $\Omega$ as follows: assume that the support of $g_{\varnothing}^{\vee}$ is contained in some closed interval $I_{\phi}$. For $\omega \in \Omega$, define the real-valued function $g_{\omega}(x)$ on $I_{\phi}$ : $g_{\omega}(x)=\sum_{k=1}^{K} f_{\phi}^{\vee}\left[x-\left(x_{k} \cos \phi+y_{k} \sin \phi\right)\right]$

where $\left(x_{k}, y_{k}\right)$ are from $\omega$, and $x \in I_{\phi}$. Notice that we are using (7) in the definition of $g_{\omega}$. Also, we define the objective real-valued function $\varphi$ on $\Omega$ :

$$
\begin{aligned}
\boldsymbol{\varphi}(\boldsymbol{\omega}) & =\left\|\mathrm{g}_{\phi}{ }^{\vee}-\mathrm{g}_{\omega}\right\|_{1} \\
& =\int_{I_{\phi}}\left|g_{\phi}{ }^{\vee}(x)-g_{\omega}(x)\right| \mathrm{dx}
\end{aligned}
$$

If $\varphi(\omega)>0$, (more accurately, if $\varphi(\omega)$ is substantially larger than our error of computing) then $\omega$ will be eliminated from $\boldsymbol{\Omega}$. Those members of $\boldsymbol{\Omega}$ who survive this test will be retested using (14) and (15) but with a different projection $g_{\varnothing}^{\vee}$ of $g(x, y)$. This elimination process is repeated until only one member is left in $\boldsymbol{\Omega}$. 


\section{EXPERIMENTS AND DISCUSSION}

We start this section with some particular examples. The convergence of our process to one solution depends on the nature of $g(x, y)$. Consider these instances:

(i) If $g(x, y)$ from (6) is formed from the cylinder (3) and parameters $\{(1,1),(2,2)\}$ as shown in

Fig 3a, then our procedure produces $\boldsymbol{\Omega}=\left\{\left[\begin{array}{ll}1 & 2 \\ 1 & 2\end{array}\right],\left[\begin{array}{ll}1 & 2 \\ 2 & 1\end{array}\right]\right\}$

This is due to the fact that both images in Fig 3a and Fig $3 b$ have the same $g_{0}^{\vee}$ and $g_{\frac{\pi}{2}}^{\vee}$. A third projection $\left(g_{\frac{\pi}{4}}^{\vee}\right)$ solves the problem.

(ii) Repeating the above example with parameters $\{(1,1),(1,2),(2,3),(3,1),(3,2)\}$, then: $x_{k}$ Parameters are: $1,1,2,3,3$;

$L_{\frac{\pi}{2}}=1,1,2,2,3$, and

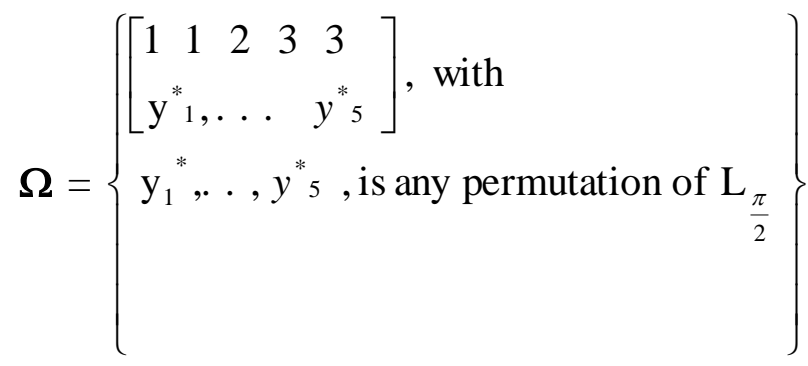

For instance, $\boldsymbol{\omega}_{1}=\left[\begin{array}{lllll}1 & 1 & 2 & 3 & 3 \\ 1 & 2 & 3 & 1 & 2\end{array}\right]$ and $\boldsymbol{\omega}_{2}=\left[\begin{array}{lllll}1 & 1 & 2 & 3 & 3 \\ 3 & 1 & 2 & 1 & 2\end{array}\right]$ are two members of $\boldsymbol{\Omega}$ that correspond to the images in Fig $4 \mathrm{a}$ and Fig $4 \mathrm{~b}$ respectively. Fig 4c, d show $g_{0}^{\vee}$ and $g_{\frac{\pi}{2}}^{\vee}$ of these images. Although one more projection was sufficient to solve the problem, we make this observation: $\boldsymbol{\Omega}$ has 120 members from which "24 solution" survived the test from (15) (using 
$g_{\frac{\pi}{4}}^{\vee}$ ). However, because the set $x_{1}, x_{2}, \ldots, x_{5}$ and $L_{\frac{\pi}{2}}$ contain repeated elements, then some of these 24 candidates are identical, some are equivalent in the sense that they appear in different order (yet they produce the same $g(x, y)$ ), and some of them are false (contains duplicated columns $\left[\begin{array}{l}x_{k} \\ y_{k}\end{array}\right]$, which can't be a solution since $g(x, y)$ is made up of non- overlapping entities). With these considerations, the " $\mathbf{2 4}$ solutions" can easily be reduced to the one true solution. (iii) Applying this procedure on the function $g(x, y)$ that was shown in Fig $2 \mathrm{a}$, we find that the calculation converge to one solution using $g_{0}^{\vee}, g_{\frac{\pi}{2}}^{\vee}$, and $g_{\frac{\pi}{4}}^{\vee}$.

Perhaps if the image presentation of $g$ from (6) is made up of entities that are surrounded by extensive regions of space, as accepted in [1],[2],[3], then the superposition back projection of $g_{0}^{\vee}$ and $g_{\frac{\pi}{2}}^{\vee}$ would produce $K^{2}$ potential peaks as already shown in figure 1 , indicating that the question of $K^{2}$ potential peaks is a trivial one. However, in a more complex situation, this superposition back-projection might fall. For instance, in the back-projection image of Figure $2 \mathrm{~d}$, it is possible that some background pixels have a higher grey level than some of the "good pixels".

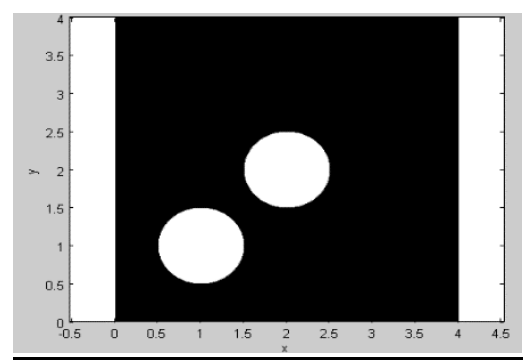

(a)

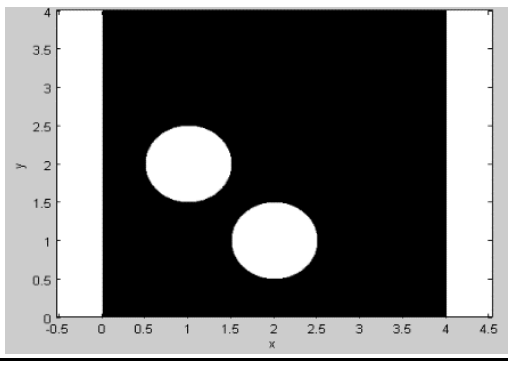

(b)

Figure 3. (a) $g(x, y)$ from (6) is formed from the cylinder (3) and parameters $\{(1,1),(2,2)\}$. (b) Possible solution since $g_{0}^{\vee}$ and $g_{\frac{\pi}{2}}^{\vee}$ are identical for both images. 


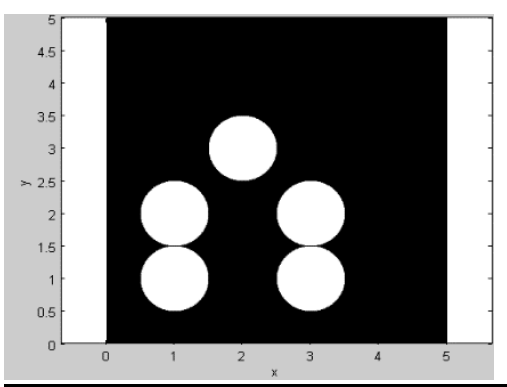

(a)

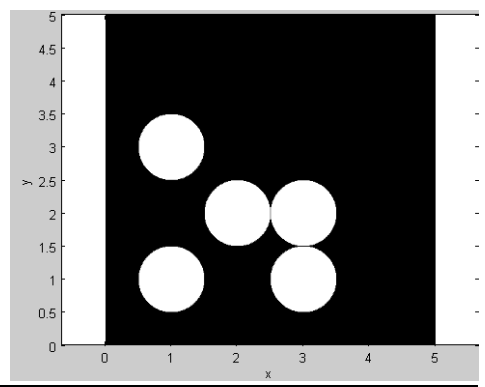

(b)

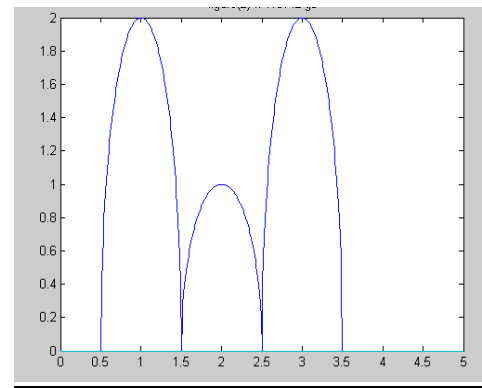

(c)

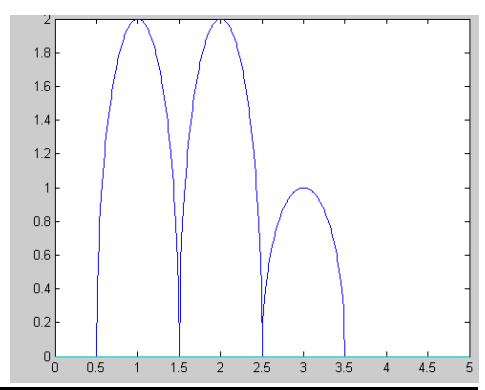

(d)

Figure 4. (a) $g(x, y)$ from (6) is formed from the cylinder (3) and parameters $\{(1,1),(1,2),(2$, $3),(3,1),(3,2)\}$. (b) A possible solution (c,), and (d) $g_{0}^{\vee}$ and $g_{\frac{\pi}{2}}^{\vee}$ for both images

\section{CONCLUSION}

In this paper, we considered a binary function $g$ formed from a binary function $f$ with compact support. The image representation of $g(x, y)$ is made up of non-overlapping translates of $f$. We introduced procedure that can recover the translation parameters using a minimum number of Radon projections $g_{\varnothing}^{\vee}$ of $g$. 


\section{REFERENCES}

[1] Kupce E and R Freeman (2004) The Radon Transform: A New Scheme for Fast Multidimensional NMR, Concepts in Magnetic Resonance, Wiley Periodicals 22: 4-11.

[2] Kup ce E, Freeman R. 2003. New Method for Fast Multidimensional NMR J. Biomol. NMR 27: 101-13.

[3] Kup ce E and Freeman (2003) Reconstruction of the three-dimensional NMR spectrum of protein from a set of projection. J. Biomol. NMR 27: 383-387.

[4] Kim S and Szyperskit (2003) GFT NMR, a new approach to rapidly obtain precise highdimensional NMR spectral information. J. Am. Chem. Soc. 125: 1385-1393.

[5] Kozminski W and I Zhukov (2003) Multiple quadrature detection in reduced dimensionality experiments. J. Biomol. NMR 26: 157-166.

[6] Frydman L, Scherf T, and A Lupulescu (2002) The acquisition of multidimensional NMR spectra within a single scan. Proc. Natl. Acad. Sci. USA 99: 15859-15862.

[7] Pelupessy P (2003) Adiabatic signal-scan two-dimensional NMR spectroscopy. J. Am. Chem. Soc. 125: 12345-12350.

[8] Ding K, and AM Gronenborn (2002) Novel 2D Triple-Resonance NMR Experiments for Sequential Resonance Assignments of Proteins. J. Magn. Reson. 156 (2): 262-268.

[9] Brutscher B (2004) Combined frequency- and time-domain NMR spectroscopy. Application to fast protein resonance assignment. J. Biomol. NMR 29 (1): 57-64.

[10] Deans SR (1983) The Radon Transform and Some of Its Applications. New York; John Wiley \& Sons, Inc.

[11] Ridge CD and VA Mandelshtam (2009) On projection-reconstruction NMR. J. Biomol. NMR 43 (3): 151-159.

[12] Coggins BE, RA Venters, and P Zhou (2010) Radial sampling for fast NMR: concepts and practices over three decades. Prog. NMR Spectrosc. 57: 381-419.

[13] Hjouj F (2011) Linear Transformation Recognition Using Radon Transform. Journal of Mathematical Sciences \& Computer Applications 2011, 1 (2): 40-47. doi:

10.5147/jmsca.2011.0038. 